\title{
ANALYSIS OF UPPER MANTLE STRUCTURE USING WAVE FIELD CONTINUATION OF $P$ WAVES
}

\author{
By Marianne C. Walck and Robert W. Clayton
}

\begin{abstract}
Wave field continuation transforms seismic record section data directly into velocity-depth space, simultaneously providing an estimate of model nonuniqueness. This inversion, previously used for reflection and refraction data, converts readily to spherical earth problems through simple adjustments in each of the two linear transformations: the slant stack and downward continuation. Because the time resolution inherent in the data transforms to depth resolution in the model space, this method is extremely useful for analysis of data compatibility with preexisting models and direct comparison between data sets, as well as the complete inversion of raw data for structure. Wave field inversion demands densely sampled, digital data, and assumes source coherency and lateral homogeneity along the profile.

We test this technique for upper mantle analysis using a previously studied, large, array-recorded data set representative of structure beneath the Gulf of California. We compare slant stacks and downward continuations of both synthetic and data record sections to illustrate the method's resolution capability. Wave field continuation proves particularly useful in comparing entire data sets to various models; even subtle structural differences are resolvable given good data quality.
\end{abstract}

\section{INTRODUCTION}

Numerous studies in the geophysical literature document the upper mantle velocity structure of tectonically interesting regions. Assembly of a detailed global map of the upper mantle would be very useful in enlarging our understanding of plate tectonics and mantle convection. Such a project requires comparison of many models derived with differing techniques and multiple data types; the uncertainties associated with the velocity-depth profiles become very important in assessing regional differences.

Quantification of the uncertainties in upper mantle models is a pervasive problem, since many of the published models were achieved through trial-and-error methods with various combinations of data constraints. One way to estimate the nonuniqueness of such models is through an extension of the trial-and-error concept: the Monte Carlo method (Wiggins, 1969). This computer intensive scheme generates a large number of random models and tests them for consistency with the data, thereby mapping an acceptable region in velocity-depth space.

If only travel-time data are available, the tau method (Bessonova et al., 1974, 1976 ) is capable of inverting large data sets and estimating the associated extremal bounds on the uncertainty. Upper mantle researchers utilizing array data (e.g., England et al., 1978; Walck, 1984a) have used this type of inversion extensively. The extremal envelope is clearly a maximum error estimate, since each envelope boundary is not itself an acceptable model. Often, additional data types such as ray parameter and amplitude information are used to constrain the models. The uncertainties in these data are not generally used in calculating the extremal bounds, although Wiggins et al. (1973) have provided a method for including ray parameter uncertainties. If still more data are included, such as waveform constraints derived 
from forward modeling with synthetic seismograms, the uncertainty envelope should be even more restrictive. Quantitative description of the uncertainty reduction obtained through this process, however, is not straightforward.

Another approach is to abandon forward modeling in favor of more formalized inversions. The series of papers by Backus and Gilbert $(1967,1968,1970)$ outlines general techniques for obtaining inverses for geophysical problems with accompanying estimates of model resolution and uniqueness. More recently, Given (1984) and Shaw (1983) develop inversion schemes based on comparisons of waveform data and synthetic seismograms. Because of high computational costs, only limited data can be included in these algorithms.

For large, densely sampled data sets, wave field continuation (Clayton and McMechan, 1981) provides direct estimates of the data uncertainty in the slownessdepth domain. The entire wave field, in the form of a record section $(T, \Delta)$ is transformed to the $(\tau, p)$, or intercept time-ray parameter, domain through the inverse Radon transform or slant stack. This process forms the $\tau-p$ curve directly, without any travel-time picks. Downward continuation with a prespecified velocity model carries the stacked data to the slowness-depth $(p, z)$ plane. Both of these processes are linear, and the final result contains all the information present in the original data set. The width of the slowness-depth image is governed by the data's coherency, quality, and inherent time resolution. Important in application of the method are the underlying assumptions of densely sampled data and lateral homogeneity (see McMechan et al., 1982 for details). Also, the presence of a velocity reversal in the generating structure will result in an offset in $\tau$ for fixed $p$. A priori knowledge of the existence of the low velocity zone is necessary to properly image it using the downward continuation process.

In previous work, wave field continuation was applied to flat-earth problems involving reflection and refraction data (Schultz and Claerbout, 1978; Clayton and McMechan, 1981; McMechan et al., 1982). Through a simple substitution this method is adaptable to problems requiring spherical geometry such as upper mantle modeling. Because of the necessity of dense spatial sampling, wave field analysis is most applicable to data collected at seismic arrays. Since seismograms from several earthquakes at various distances from an array are required to assemble an upper mantle record section, we apply some preprocessing of the data to simulate a single source. Static shifts due to receiver structure are removed, and source wavelet equalization is attempted through a simple deconvolution procedure. We test the wave field technique's usefulness for upper mantle data with analysis of synthetic record sections, and then compare the synthetic results to those for real data representing Gulf of California upper mantle structure.

\section{THEORY}

Wave field continuation consists of two linear transformations of the data: the slant stack and downward continuation (see Clayton and McMechan, 1981, for a detailed discussion). The slant stack transforms a seismic record section $P(t, x)$ from the $(t, x)$ domain to the $(\tau, p)$ domain through the relation (e.g., McMechan and Ottolini, 1980)

$$
S(\tau, p)=\int_{-\infty}^{\infty} P(\tau+p x, x) d x
$$

$S$ is the slant stacked wave field; $\tau$ is the delay time, $T-p x ; t$ is travel time; $x$ is 
distance, and $p$ is ray parameter. By decomposing the wave field into its plane wave components, this process forms an image corresponding to the tau curve of Bessonova et al. (1974, 1976). In stacking the data, we assume lateral homogeneity along the profile and require sufficient spatial sampling to prevent aliasing. Coherent source signatures are also important in obtaining a satisfactory image.

The next step is downward continuation of the $(\tau, p)$ data to the $(p, z)$ domain $(z$ is depth) using a prescribed velocity model $v(z)$. For Cartesian geometry, the slowness-depth image, $s(p, z)$ is

$$
s(p, z)=S(\tau=0, p, z)=\int S(\omega, p, 0) \exp [-i \omega \Psi(p, z)] d \omega
$$

where $\omega$ is frequency, and

$$
\Psi(p, z)=2 \int_{0}^{z}\left[\frac{1}{v(z)^{2}}-p^{2}\right]^{1 / 2} d z
$$

Notice that (3) is simply the definition of $\tau(p)$. The above equations show how the wave field at any depth is formed from the known stacked field at the surface, $z=$ 0 . An approximate form of (2) in the time domain, is

$$
s(p, z)=S[\Psi(p, z), p, 0] .
$$

Adaptation of the method to a spherical Earth is straightforward. One valid approach is to change the spherical model and data to the equivalent Cartesian form using the standard earth-flattening approximation (e.g., Müller, 1971). After invoking (1) and (4), the resulting $(p, z)$ wave field is transformed back to its spherical counterpart $[p(r), r]$, where $r$ is radius. We can circumvent the earthflattening approximation and achieve the same result by substituting the spherical version of $\tau(p)$ in equation (3)

$$
\Psi(p, r)=2 \int_{r_{0}}^{r}\left[\frac{1}{u(r)^{2}}-\frac{p^{2}}{r^{2}}\right]^{1 / 2} d r
$$

in the inversion process. Here, $u(r)$ is spherical velocity, and $r_{0}$ is the Earth's radius. The teleseismic data are first stacked in linear $p(r)$ to take $(t, \Delta)$ to $[\tau, p(r)]$ and then downward continued according to

$$
s(p, r)=S[\Psi(p, r), p, 0] .
$$

Since the continuation step requires an initial velocity model, $u(r)$, an inversion from scratch is an iterative process. Convergence is achieved when the specified model coincides with the generated wave field. Another application of this technique is in a comparative sense: we can verify the suitability of an existing velocity model for a data set, or compare two different groups of data by continuing them both relative to some reference model. Comparisons can also be made in the $(\tau, p)$ domain, but the downward continuation process yields additional information on depth resolution.

A previously analyzed, densely spaced upper mantle data set for the Gulf of 
California (Walck, 1984a) provides an excellent example for demonstration of this technique. The results of wave field continuation of these data and a synthetic section generated from the postulated velocity model, GCA, are presented in the next section.

\section{Application: Synthetic and Data Examples}

The ideal upper mantle data profile for wave field continuation inversion ranges to beyond $30^{\circ}$ epicentral distance, is digital, well-sampled spatially with no gaps in coverage, and has simple, coherent source functions throughout the distance range. Most data sets do not meet all these specifications; one that approaches closer than most is the 1363-seismogram profile of Walck (1984a) recorded from Mexican

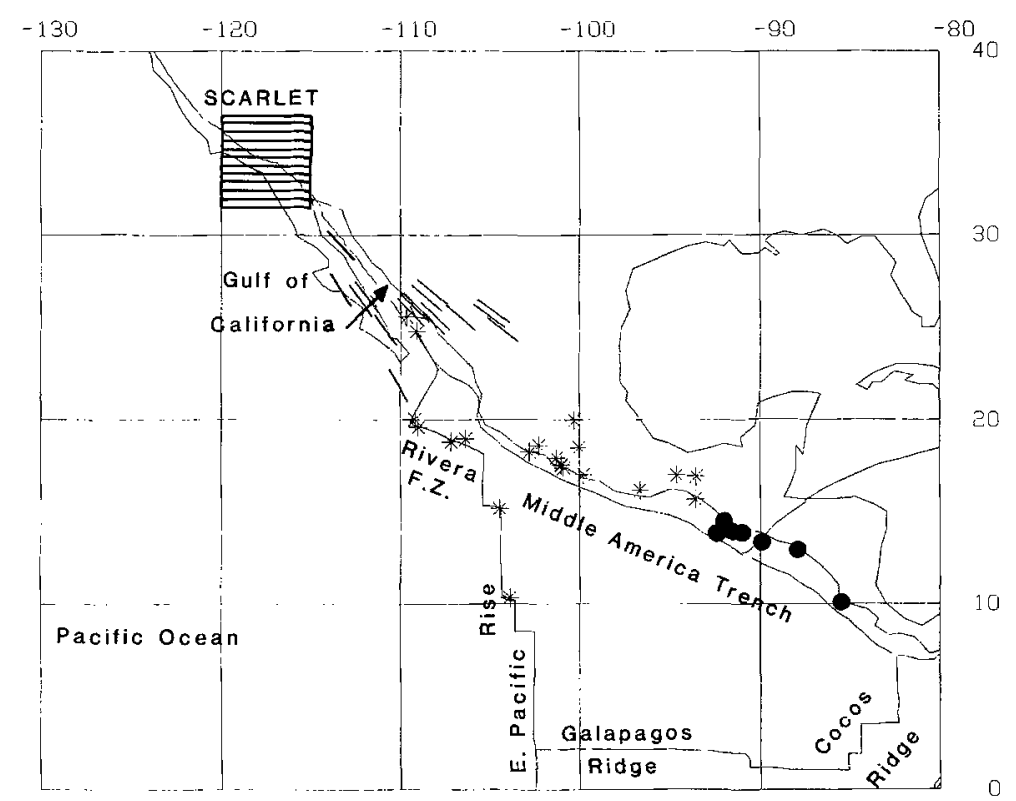

FiG. 1. Location map for the 29-event data example presented in the Application section. Stars represent 22 events in the distance range $9^{\circ}$ to $30^{\circ}$ from southern California; dots are seven events farther than $30^{\circ}$. Each event is recorded over a $4^{\circ}$ to $5^{\circ}$ swath of distance by SCARLET, the 200-element southern California network telemetered to Pasadena. Short line segments are $2^{\circ}$ arcs drawn at the midpoints of each event's great-circle path to Pasadena, indicating the extent of the data coverage.

earthquakes at the 200 station Caltech-USGS Southern California Seismic Network. The 29 events cover a distance range of $9^{\circ}$ to $40^{\circ}$ and represent the upper mantle beneath western Mexico and the Gulf of California (Figure 1). Earthquake mechanisms in this region vary from strike-slip events in fracture zones to northweststriking thrusts in the Middle America Trench, so we do not achieve source coherency along the profile. The many available records do, however, continually cover the entire $31^{\circ}$ distance range except for small gaps near $14^{\circ}$ and $19^{\circ}$. The total azimuthal variation of the profile is less than $30^{\circ}$. Figure 2a shows a 373record data subset containing the simplest 10 events which span the entire range. The additional data are primarily from distances greater than $20^{\circ}$, already wellcovered in this smaller suite of records.

Using a combination of travel time, $d T / d \Delta$ and waveform data, Walck (1984a) derived a model, GCA, to fit these spreading center data. GCA features very slow velocities to $350 \mathrm{~km}$ depth, consistent with upwelling of hot material beneath the 
ridge. An unusually steep velocity gradient from 225 to $390 \mathrm{~km}$ brings the model into close agreement with published models for depth greater than $400 \mathrm{~km}$. GCA has a $7.9 \mathrm{~km} / \mathrm{sec} P n$ velocity and a small low-velocity zone; it is actually appropriate not for the ridge but for continental regions adjacent to the Gulf of California. To fit travel times for events $\left(9^{\circ}<\Delta<13^{\circ}\right)$ occurring within the Gulf itself, GCA', which has no low-velocity zone and features a gradual crust-mantle transition, was constructed. Throughout this discussion, we use the model GCA'-GCA, which consists of $\mathrm{GCA}^{\prime}$ to $150 \mathrm{~km}$ depth underlain by GCA (Figure 3). Figure $2 \mathrm{~b}$ is a WKBJ synthetic seismogram profile of $\mathrm{GCA}^{\prime}$-GCA.

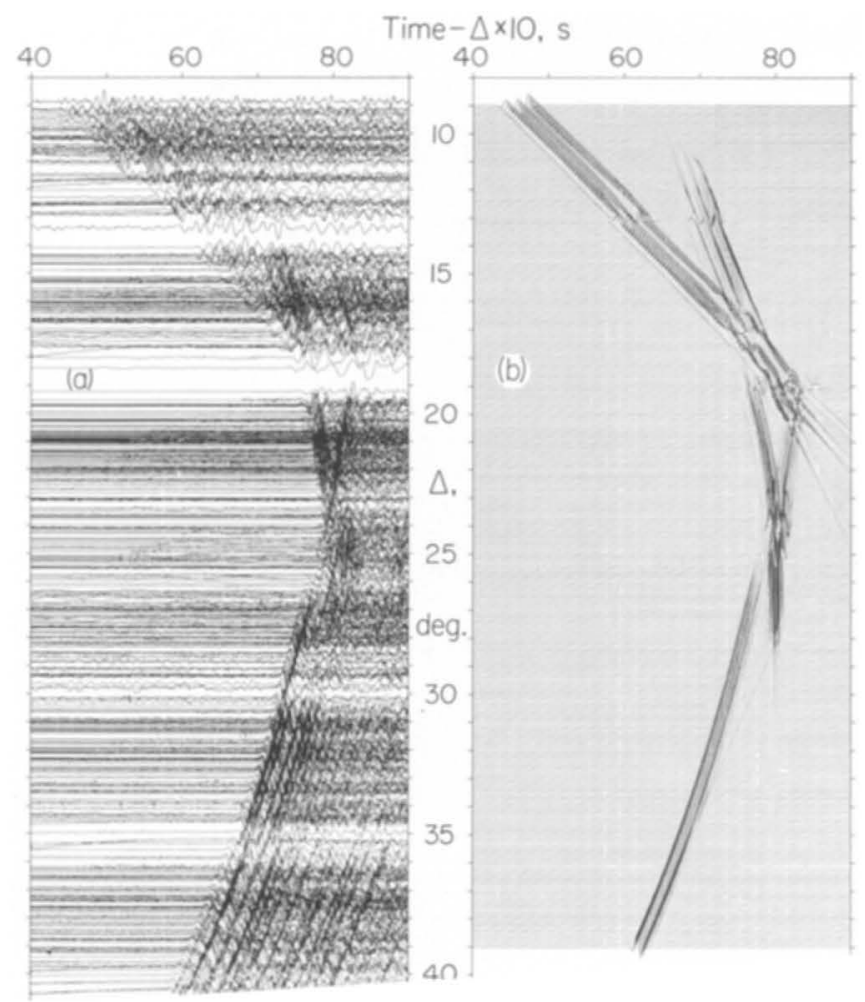

FIG. 2. Data and synthetic record sections for the Gulf of California data. (a) A 10-event, 373-record data subset covering $9^{\circ}$ to $40^{\circ}$. The seismograms are plotted at their surface focus-corrected distances and have been filtered, corrected for receiver statics and for uniform polarity. (b) Synthetic profile for models $\mathrm{GCA}^{\prime}\left(\Delta \leqq 13^{\circ}\right)$ and $\mathrm{GCA}\left(\Delta>13^{\circ}\right)$ covering the same distance range. Three source wavelets are used, varying with distance along the profile.

We utilize a noise-free synthetic record section to test the spherical version of the wave field continuation inversion and to determine the maximum resolution of our short-period $P$ waves. First the record section is stacked, then downward continued with the generating model, GCA'-GCA. The continued synthetics should coincide with the input model, and the width of the image gives the ideal depth resolution. Next, the earthquake data are inverted in the same fashion, again using $\mathrm{GCA}^{\prime}$-GCA as the velocity model. If this model is consistent with the data, the image will match $\mathrm{GCA}^{\prime}$-GCA in $(p, r)$ space, again with some uncertainty envelope. GCA was formulated through a complicated procedure involving Bessonova et al.'s $(1974,1976)$ tau inversion, ray parameter constraints, and forward modeling using synthetic seismograms. The limits of nonuniqueness, therefore, are not well known. 
The continuation of the data with GCA'-GCA will yield estimates of the resolving power of the actual data, and thus the model uniqueness. A further application is comparison between different models and the real data. In either the $(p, \tau)$ or $(p$, $r$ ) domain, the compact representation of the stacked or continued data makes a "global" comparison easy between models and data or between several data sets.

Data preparation. Although timing of phases is not necessary for wave field inversion, the source incoherency and static variations associated with earthquake data make some preprocessing desirable for an optimal image. We remove static receiver variations through a simple least-squares averaging procedure. Timing of first arrivals for several "calibration events" from the same azimuth $\left(\Delta>30^{\circ}\right.$; see Figure 1) is required. Deviations from the least-squares plane fits to these times are averaged over several events; records from stations with inconsistent residuals are

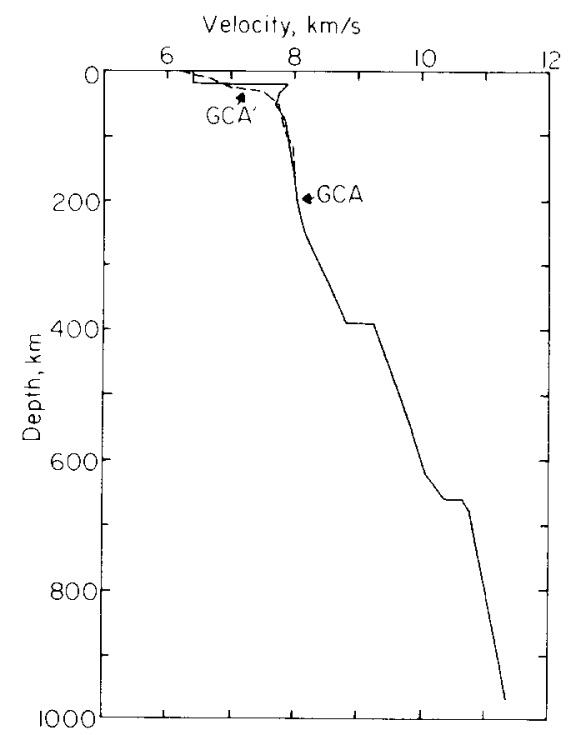

FIG. 3. Models GCA' and GCA. GCA', which extends to $175 \mathrm{~km}$ depth, is appropriate for the axis of the Gulf of California, while GCA is more fitting for adjacent continental areas. The synthetic examples are generated using the combined model $\mathrm{GCA}^{\prime}-\mathrm{GCA}$, which uses $\mathrm{GCA}^{\prime}$ for the top $150 \mathrm{~km}$ and GCA beneath.

discarded. The resulting station corrections include effects of elevation and receiver structure, and are useful for only a limited ray parameter-azimuth window. Alignment of individual record sections dramatically improves after application of these corrections (see Figure 8 in Walck, 1984a). Since some of the events occur in the mantle, we apply both time and distance adjustments for each event depth. The 10event composite record section, Figure $2 a$, shows a subset of the filtered data corrected for depth and receiver structure. While further improvement would accompany removal of static effects caused by source mislocations, the alignment is excellent. We did not apply any source-related baseline shifts.

The data are filtered with a low pass filter to suppress high frequency $(>5 \mathrm{~Hz})$ noise. Since the spectra of the events are quite band-limited with a central frequency near $1 \mathrm{~Hz}$ (Figure 5, middle column), the filtering leaves the signal unaffected.

The interesting upper mantle phases all arrive within about $10 \mathrm{sec}$ of the first wave motion on each record. In order to window out large, noisy coda waves such as those caused by crustal reverberations and also to eliminate $p P$ phases from 
deeper events, we apply a 5 -sec cosine taper commencing after the initial 10 sec of signal.

The key ingredients in obtaining a good stacked image are consistent timing, polarity, and source coherency. The steps outlined above help in lining up phases, and phase polarities are easily adjusted to be uniform. Figure 4 demonstrates the destructive effect of source variation along the profile. Shown are two $(\tau, p)$ plots

(a)
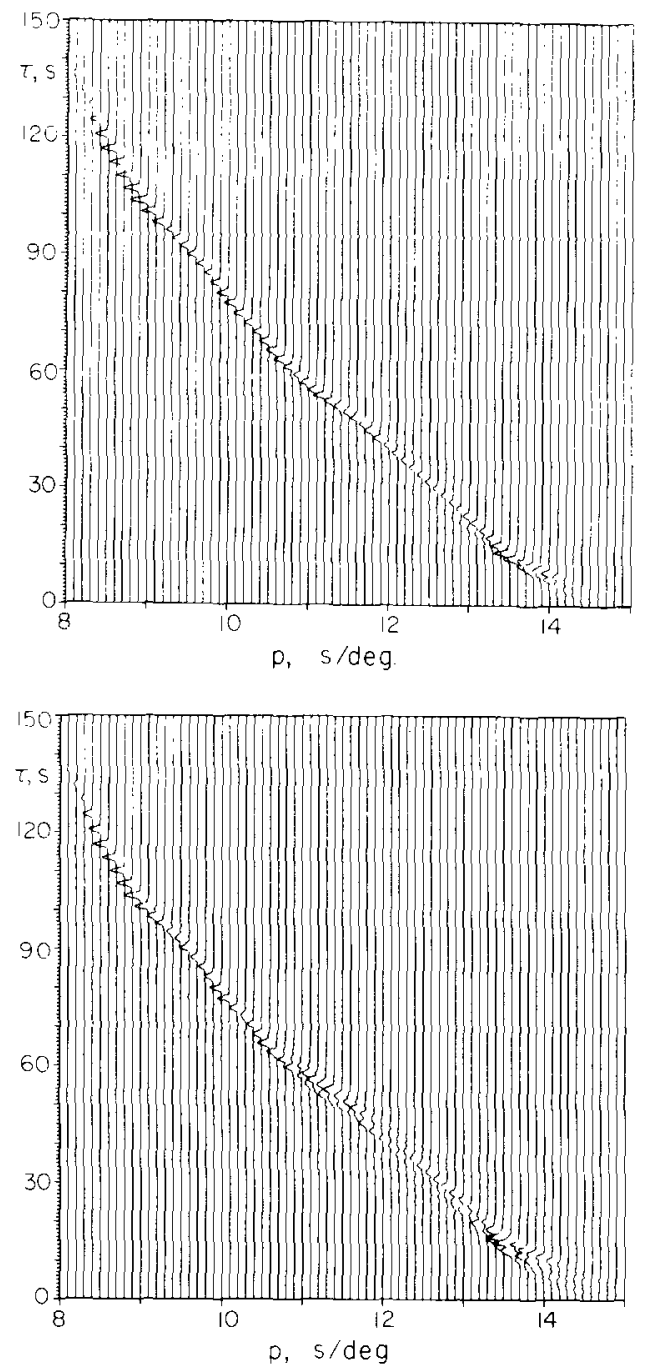

FIG. 4. Two slant stacks of synthetic data generated with GCA'-GCA. (a) Stack for a single-source profile. For each value of $p$, we plot the envelope of the stacked wave field. (b) Same for the varyingsource profile of Figure $2 \mathrm{~b}$. Notice the poorer image resolution for ray parameters near 11 and 13 to 14 $\mathrm{sec} / \mathrm{deg}$.

of stacked synthetic wave fields generated with $\mathrm{GCA}^{\prime}$-GCA synthetics. The envelope function of the stack is plotted for each $p$ value. For the one-source profile, Figure $4 a$, the tau image is clear. The introduction of only three different sources into the noise-free synthetics, however, produces the image degradation observed in Figure 4 b. For the multiple sources encountered in the real, noisy data set, we expect still poorer image quality. 
In an attempt to equalize the source wavelets between earthquakes, we apply a simple, clamped frequency division deconvolution to each record. The source estimate for each event is obtained from an array-recorded seismogram for which the first-arriving wave turned in a smooth portion of upper mantle, producing a pulse which consists of the convolution of the instrument response and the source-time function. The array instrument responses are all similar at teleseismic frequencies. Since each event is recorded over a (nonnodal) limited azimuthal range, the earthquake's radiation pattern remains relatively constant across the array and a

$\begin{array}{ccc}\text { Source } & \text { Source } & \text { Raw } \\ \text { Wavelet } & \text { Spectrum } & \text { Data }\end{array}$
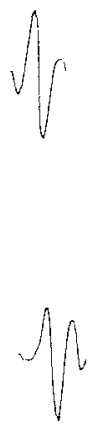

$5 \mathrm{~s}$

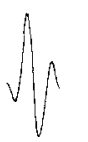

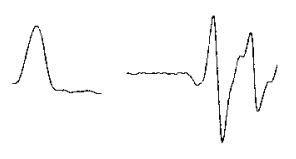

3.125 Hz CPM

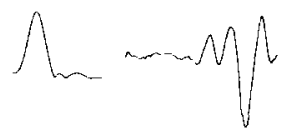

SBLC

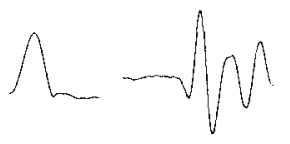

CPM $\begin{array}{lr}\text { Deconvolved Data } \\ w=0.5 & w=0.1\end{array}$
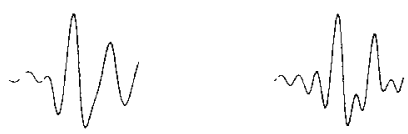

$21.8^{\circ}$
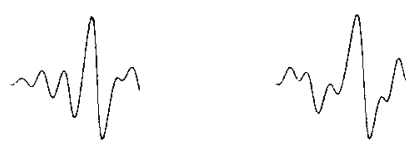

$23.1^{\circ}$
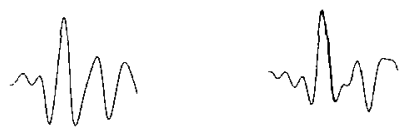

$28.0^{\circ}$

FIG. 5. Examples of clamped frequency division deconvolution. Each row contains, for one event, the source wavelet, source spectrum, data trace, and two deconvolved versions of the data using different values of $w$, the damping parameter. As $w$ decreases, the deconvolved data look less like the original data, becoming more minimum phase and spikelike in character. In the first row, the raw data contains both the first arrival and a reflection from the $670 \mathrm{~km}$ discontinuity $4 \mathrm{sec}$ later. The deconvolved version with $w=0.1$ shows the double arrival very clearly. The second row data show an interference pattern between the first arrival and the $670 \mathrm{~km}$ discontinuity reflection. The second deconvolved trace distinguishes the first arrival better than the first. Data traces from station CPM are shown for the first and third events and SBLC for the middle row. The source wavelets are from various stations, depending on the event. Most source spectra are smooth and peaked at 0.7 to $0.8 \mathrm{~Hz}$. Records shown here are typical results, not the best examples.

single source wavelet characterizes each event. We deconvolve every record with the appropriate source wavelet. Samples of typical deconvolution results at two stations appear in Figure 5. The complexity of the deconvolved waveforms is reduced, and the new wavelets are more minimum phase in character.

Finally, we deal with the problem of unequal trace spacing by interpolation of the filtered, deconvolved, and tapered seismograms to equal distance intervals of $0.1^{\circ}$. The resampling (interpolation) is accomplished by a weighted sum of all traces within $0.2^{\circ}$ of one of the resampled seismograms. The weights are inversely proportional to the distance from the resampling point and are normalized to a unity sum. The resampling interval in this case is $0.1^{\circ}$. This record spacing is not sufficient to avoid all spatial aliasing, but is convenient for comparison with the synthetic section generated at the same sampling interval. This obvious approximation is an alter- 
$T$ - Delta $\times 10, \mathrm{~s}$

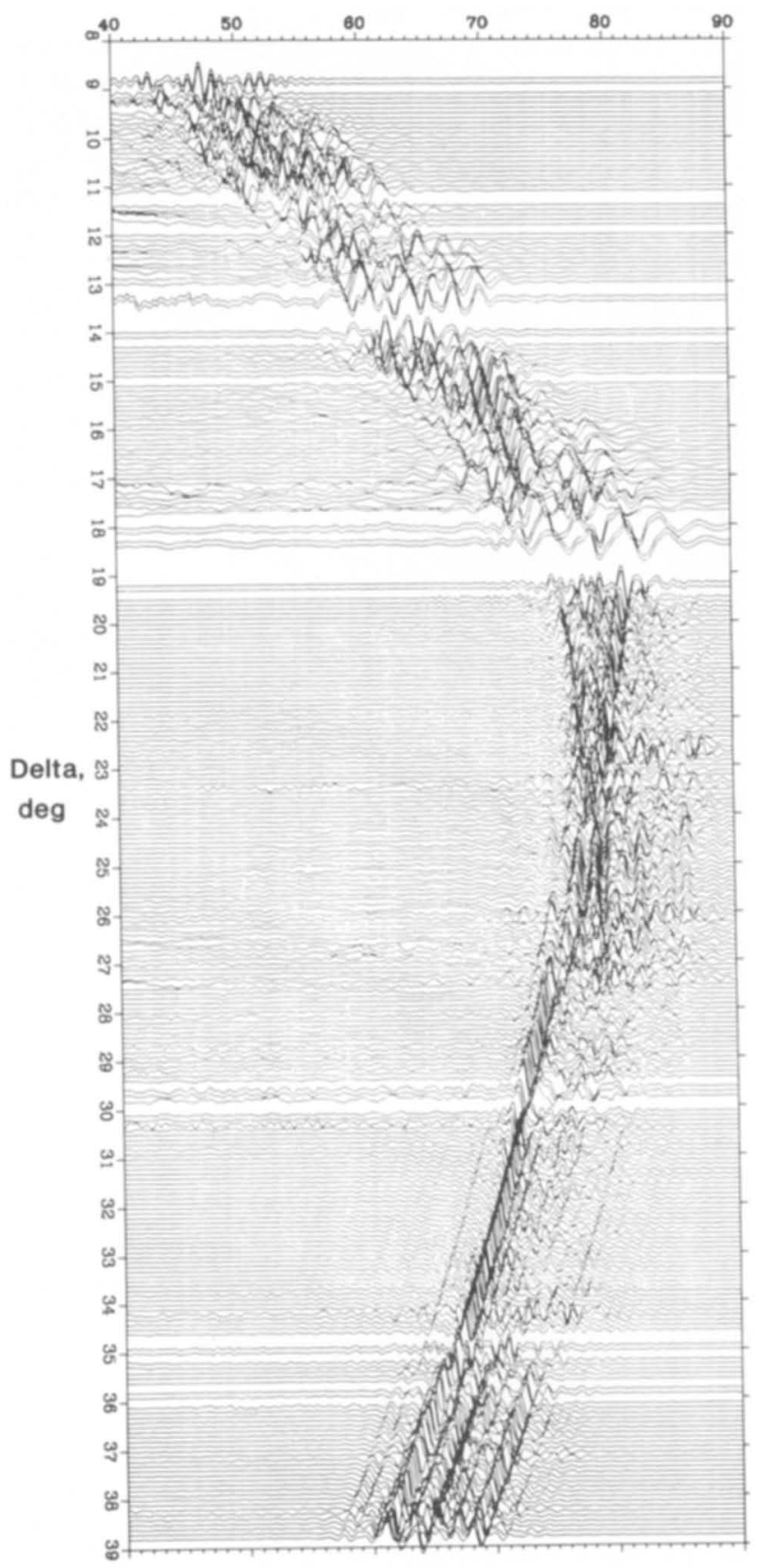

FIG. 6. The 10-event data set after processing described in the text. These are the same data as in Figure $2 \mathrm{a}$ after deconvolution, cosine tapering, and resampling to $0.1^{\circ}$ distance increments. The final data set contains 279 seismograms.

native to weighting the slant stack so that distance ranges containing more data do not predominate the tau image. After processing as outlined above, the 10 -event data set of Figure 2a is resampled to 279 seismograms from $9^{\circ}$ to $39^{\circ}$ and appears in Figure 6. These data, along with a deconvolved, single-source version of the 301- 
record synthetic record section shown in Figure $2 b$, are ready for the stacking and downward continuation transformations.

Slant stacks. An image in the $(\tau, p)$ domain emerges from the data after application of the inverse Radon transform (1) to a seismic record section. Because our data are distributed in "upper mantle distances", $9^{\circ}$ to $40^{\circ}$, a $p$ range of 8 to $15 \mathrm{sec} / \mathrm{deg}$

(a)

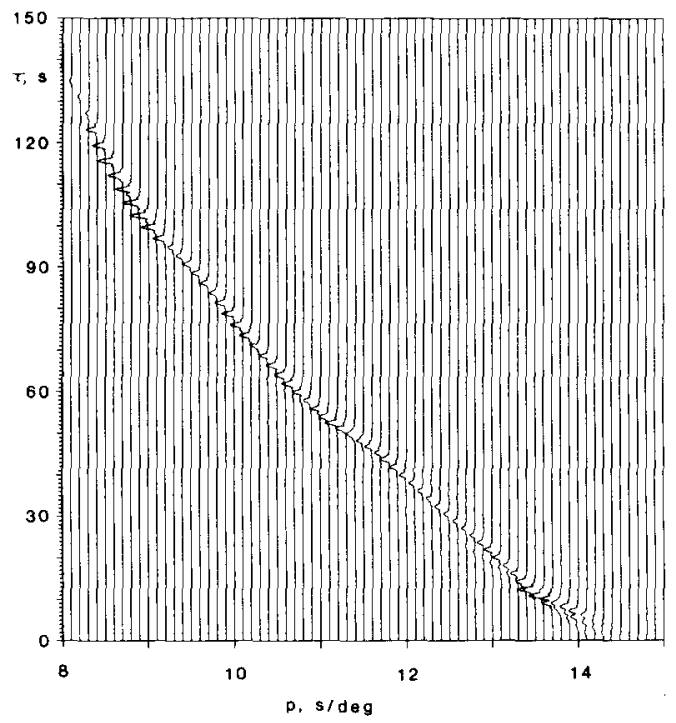

(b)

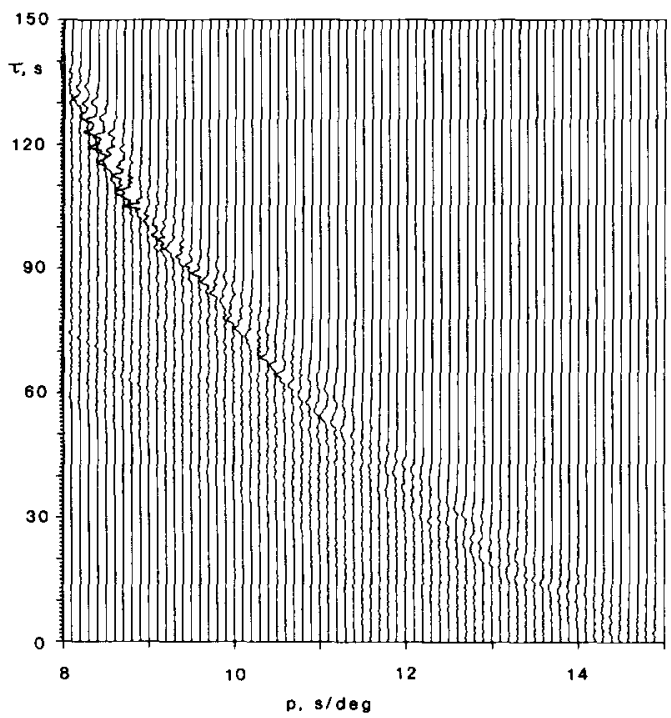

(c)

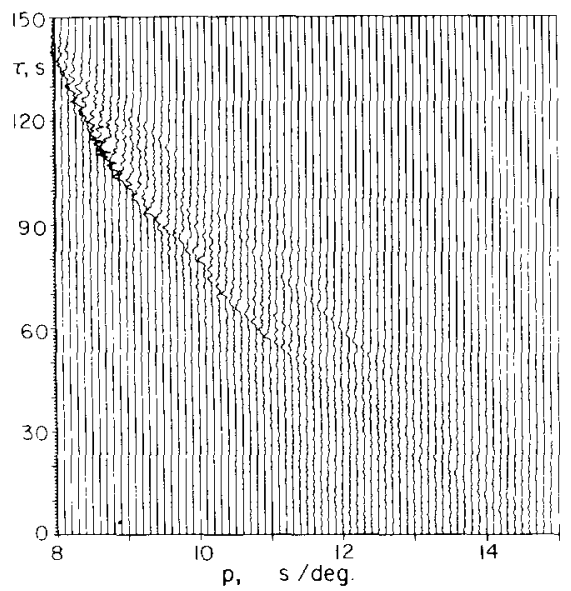

FIG. 7. Stacked wave fields of synthetic and data profiles. (a) GCA'-GCA synthetic stack. Same as Figure 4a except that the synthetic records are deconvolved in the same manner as the data. (b) Stack of the "processed" data shown in Figure 6. (c) Same for the "unprocessed" data of Figure 2a. Notice the noisier image.

is used with a ray parameter spacing of $0.1 \mathrm{sec} / \mathrm{deg}$; the corresponding $\tau$ values are 0 to $150 \mathrm{sec}$ in $0.08 \mathrm{sec}$ increments. Figure 7 displays the envelope function of both the synthetic and actual data stacks. The image obtained from stacking the deconvolved single-source synthetic record section (Figure 7a) demonstrates clearly how the stacking process "unravels" travel-time triplications; the relative strengths of 
multiple mantle phases are also preserved in the amplitude of the stacked waveforms. Strong first arrivals from $30^{\circ}$ to $39^{\circ}$, for example, correspond to high stack amplitudes for ray parameters of 8.4 to $9.0 \mathrm{sec} / \mathrm{deg}$. The record section ends at $39^{\circ}$, to $p$ values of less than $8.4 \mathrm{sec} / \mathrm{deg}$ have relatively little power. The same phenomenon is visible at the opposite end of the stack, where data from closer than $9^{\circ}$ would be needed to provide information about ray parameters greater than $14.1 \mathrm{sec} /$ deg.

After stacking, the 10-event processed data of Figure 6 appear as in Figure $7 \mathrm{~b}$. The data are noisier than the synthetics, but the general similarity is striking. Not only do the $(\tau, p)$ peaks coincide, the stack amplitude pattern seen in Figure 7a is reproduced in the data, at least for small $p$ values. The tau image is quite coherent for $p<11.5 \mathrm{sec} / \mathrm{deg}$, at which point the signal loses power relative to noise. This degradation is due to the less coherent regional data collected from strike-slip earthquakes at distances of $9^{\circ}$ to $14^{\circ}$. The excellent data from $20^{\circ}$ to $39^{\circ}$, on the other hand, transform to a very cohesive image for $8 \mathrm{sec} / \mathrm{deg}<p<11 \mathrm{sec} / \mathrm{deg}$. The stacked representation of the data makes it easy to see the strengths and weaknesses of an entire data group relative to the ideal synthetic case. For comparison, a stack of the unprocessed data (see Figure 2a) is shown in Figure 7c.

In order to see fine details of the slant stacks, we plot them in "reduced $\tau$ " format (Figure 8). These stacks have been adjusted relative to the arbitrarily chosen line $\tau$ $=-21.667 p+130$. The undulations in the tau curve due to upper mantle discontinuities are enhanced in this representation, and the relative amplitude changes are more obvious. The data, shown in Figure $8 \mathrm{~b}$, are again not as impressive as the synthetic stack (Figure 8a); the data amplitudes lessen dramatically for $p>11.5$ $\mathrm{sec} / \mathrm{deg}$ even where the predicted amplitudes are large, and a probable artifact appears from 13.4 to $13.9 \mathrm{sec} / \mathrm{deg}$ with $\tau$ values of 2 to $9 \mathrm{sec}$. Notice, however, that the low stack amplitudes present in the data for ray parameters between 12 and 13 $\mathrm{sec} / \mathrm{deg}$ are in accordance with the synthetic version. Figure $8 \mathrm{c}$ is an overlay comparison of the slant stacks of the data and synthetic record sections. The agreement is excellent, especially for the lower ray parameters, which correspond to depths of greater than $400 \mathrm{~km}$. Our increased uncertainty of shallow (0 to 250 $\mathrm{km}$ ) structure is reflected in the less convincing $\tau$ image for the correspondingly higher $p$ values. The overall agreement does indicate that $\mathrm{GCA}^{\prime}$-GCA is truly consistent with the Gulf of California data set as a whole.

Downward continuation. The next transformation carries the stacked data and synthetics of Figure 7 into the $(p, r)$ domain. The downward continuation calculations are done using (5) and (6), with a Simpson's rule integration and a depth step of $2.5 \mathrm{~km}$. Downward continuation of the GCA'-GCA synthetic stack with GCA'GCA produces a slowness-depth image that coincides with the input model, as expected (Figure 9a). The width of the image in Figure 9 shows how the envelope of a 1-sec $P$-wave source wavelet transforms to an indication of depth resolution. The width of the $(p, r)$ envelope is about $50 \mathrm{~km}$. If we assume that we can accurately pick the onset of the arrival to $\frac{1}{4}$ wavelength or less, the realistic resolution in depth for these data is on the order of $12 \mathrm{~km}$.

Continuation of the actual Gulf of California data with $\mathrm{GCA}^{\prime}-\mathrm{GCA}$ results in the plot of Figure 9b. The observed image again coincides with the input model, indicating consistency. This result is not surprising, since the stacked data compare well with the stacked synthetics, and both stacks are continued with the same model. The wider, more "smeared" image shows the decreased resolution obtainable from the real data. As in the $(\tau, p)$ domain, we obtain a good slowness-depth image for $p<11.5 \mathrm{sec} / \mathrm{deg}$ and poor to no resolution above the corresponding depth. When 

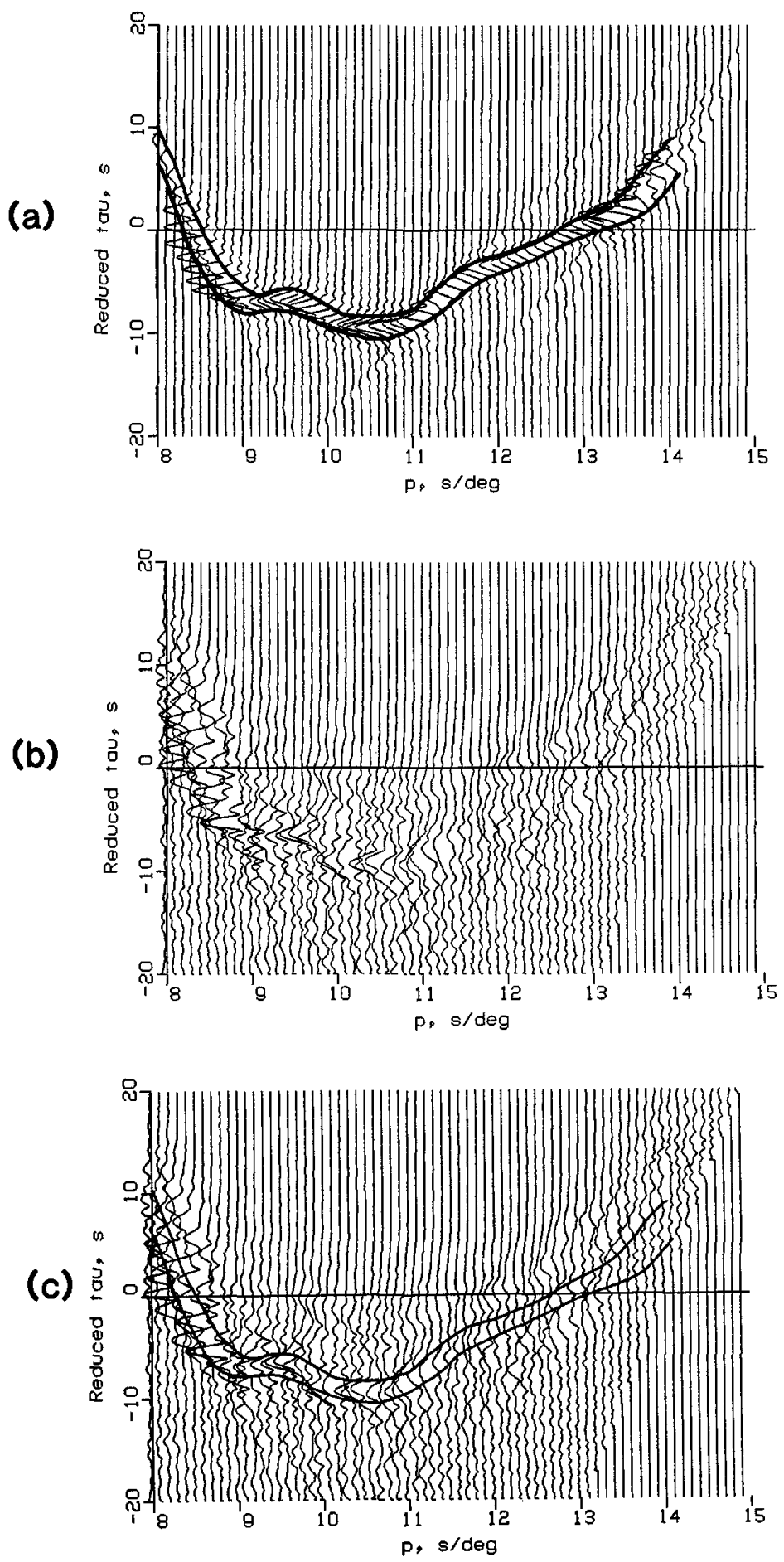

FiG. 8. "Reduced $\tau$ " slant stacks. These data are reduced relative to $\tau=-21.667 p+130$. Subtle variations in relative amplitudes are easier to see in this format. (a) GCA'-GCA synthetics. The lines enclose the region of large stack amplitudes. (b) Reformatted data of Figure 7c. Note increased noise level relative to the synthetic stack. The relative amplitude patterns are similar. (c) Same as (b) with the $\tau$ locus of (a) superimposed. The agreement is quite good. 
the input model is incorrect, the image forms far away from the model, as is demonstrated in Figure 9c. The limited number and fairly poor quality of the closer data preclude strong statements about the very shallow depths beneath the Gulf of California using this method. The downward continuation process does yield a data representation that emphasizes which portions of the model are well constrained.

(a)

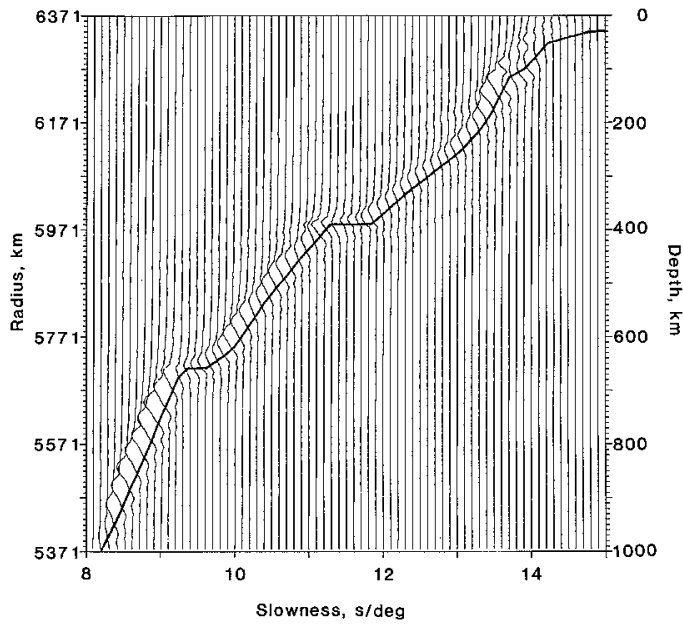

(b)

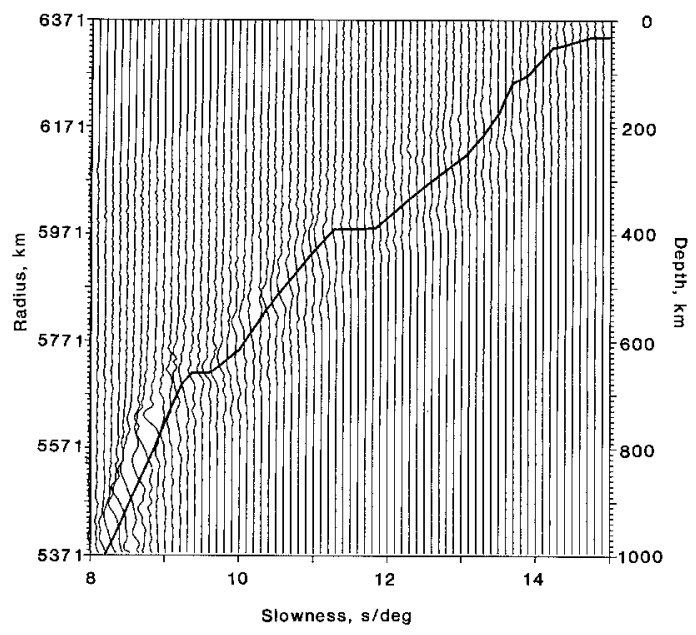

(c)

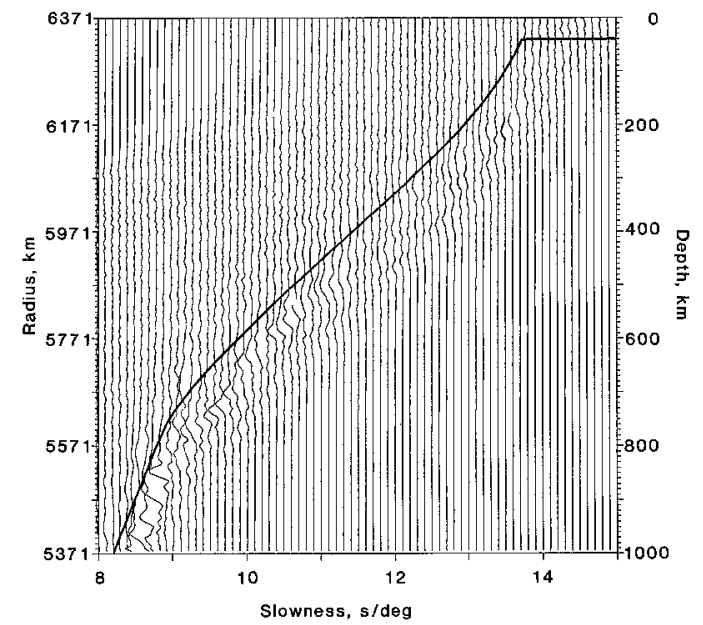

Fig. 9. Downward continuations of the slant stacks shown in Figures 7 and 8. (a) Synthetic test case. GCA'-GCA synthetic seismograms are stacked and continued with the generating model. The solid line is $\mathrm{GCA}^{\prime}$-GCA. Notice that the continued wave field coincides with the model, as expected. An indication of the depth resolution for each $p$ value is given by the width of the continued image. Even synthetic data have an uncertainty of nearly $50 \mathrm{~km}$. (b) Same for the Mexico data. The image is not as sharp, but GCA'-GCA is still clearly consistent with the data. (c) The data continued with the Herrin (1968) model (solid line). Note that this smoother model is clearly not appropriate for our data; the image falls consistently below the line.

Model comparison. In addition to providing objective estimates of model uniqueness, wave field continuation is potentially useful for comparison of entire data sets to each other and to corresponding models. By inspecting the wave fields in either the $(\tau, p)$ domain or after continuation relative to a reference model, even subtle disparities between models may be verified as resolvable. 
Data recorded at the Southern California Network from earthquakes along the northeast rim of the Pacific Ocean provide a data set for comparison with the Gulf of California records. The overall quality of these data, which represent the upper mantle beneath the Cascade Range and the Juan de Fuca plate, are poorer, and the resulting slant stack, resulting from the transformation of 269 processed seismograms covering almost $33^{\circ}$, is messy and difficult to interpret. The closer events ( $\Delta$ $<30^{\circ}$ ) from this azimuth have similàr strike-slip mechanisms which produce complicated waveforms. The calibration events $\left(\Delta>30^{\circ}\right)$ are dip-slip events near the Aleutian Trench. Walck (1984b) used GCA as a starting model for synthetic seismogram modeling of these data and derived a new model, CJF (Figure 10), which differs from the southern model only at points demanded by specific data groups. A complete discussion of the two data sets will appear in a separate

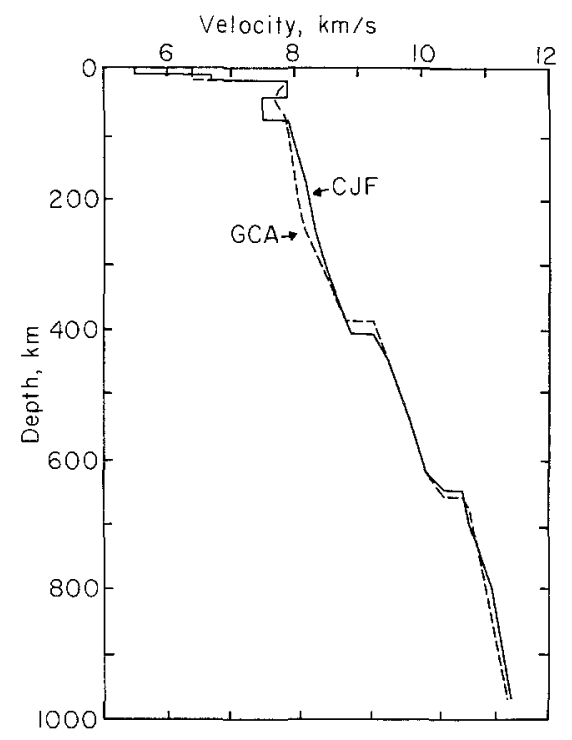

FIG. 10. Models GCA and CJF. CJF was derived for the northeast Pacific rim region in a comparative fashion using synthetic seismogram modeling with GCA as a starting model. The two models are quite similar, especially below $450 \mathrm{~km}$ depth.

publication. As Figure 10 shows, CJF is quite similar to GCA below $350 \mathrm{~km}$ except for slight differences in the discontinuity depths. The discrepancies above $350 \mathrm{~km}$ result primarily from differing travel times for regional events. The energy from these closer earthquakes, unfortunately, is quite incoherent and does not produce a good stacked image for large ray parameters (Figure 11b). Thus, direct comparison between the two data sets using wave field continuation is difficult. If we assume, however, that CJF is an adequate representation of the northeast Pacific data, we can ignore the inconclusive stack of the northern data and compare the CJF synthetics directly to the Mexican data in either the $(\tau, p)$ or $(p, r)$ domain. Such an application of the method is particularly attractive in comparing one data set to a number of published models. In Figure 11, we compare a synthetic stack for CJF with the northeast Pacific data and the Mexico data. The primary differences between CJF and GCA'-GCA (see Figures 10, 11b, and 8a) are near $660 \mathrm{~km}$ and above $450 \mathrm{~km}$; the corresponding ray parameter values are approximately 9.2 to 9.8 $\mathrm{sec} / \mathrm{deg}$ and higher than $11 \mathrm{sec} / \mathrm{deg}$. Comparison of Figures $11 \mathrm{c}$ and $8 \mathrm{c}$ reveals that for $9.2<p<9.8 \mathrm{sec} / \mathrm{deg}$, CJF predicts $\tau$ values that are too low (Figure 11c) while $\mathrm{GCA}^{\prime}$-GCA (Figure 8c) is correct. This indicates that the lower discontinuity 
(a)

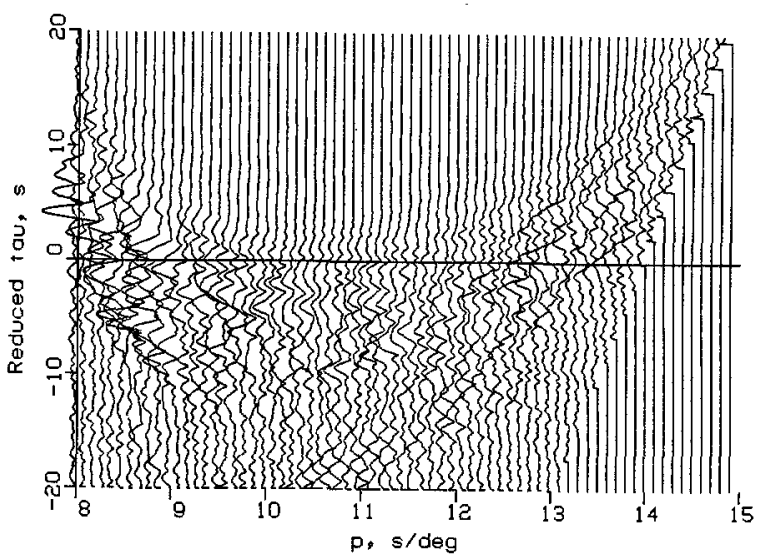

(b)
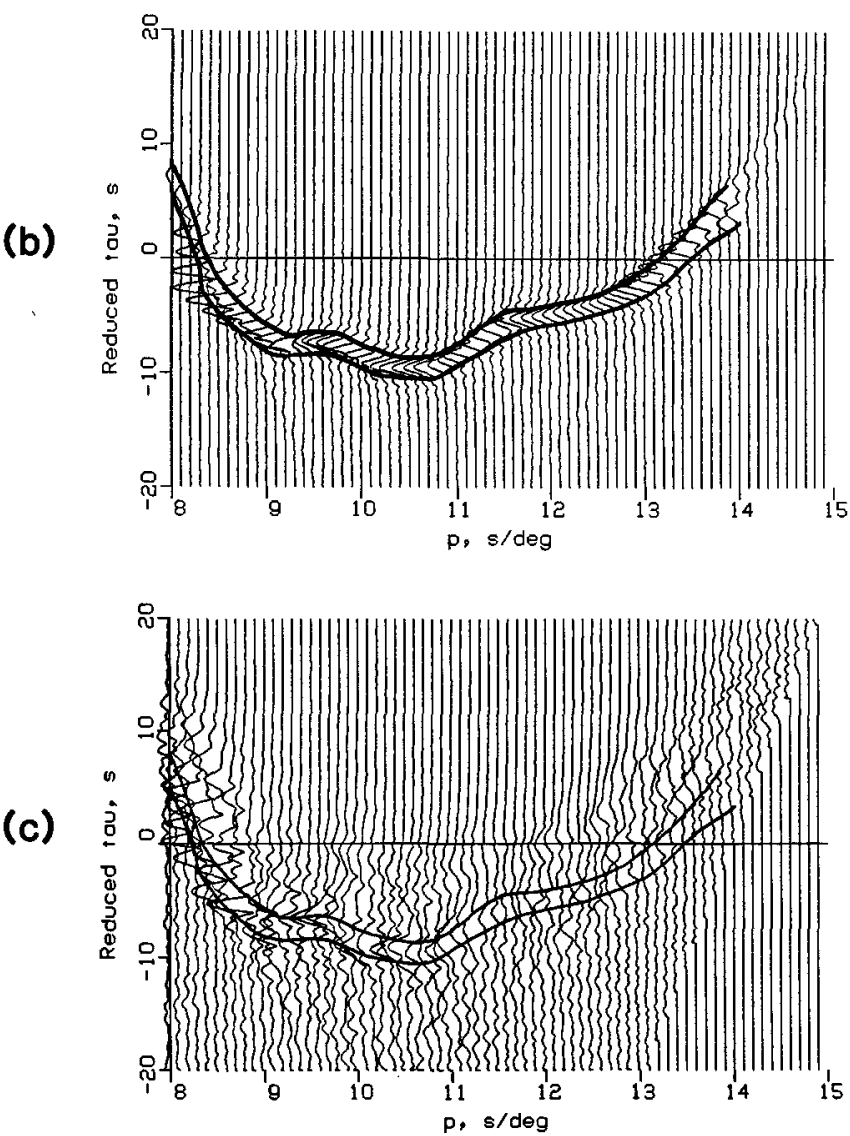

FIG. 11. Model comparison using slant stacks. (a) Stack of 269 processed records from 11 northeast Pacific events recorded at SCARLET. Notice that these data are noisier than the corresponding Mexican events (Figure $8 \mathrm{~b}$ ). Large linear artifacts are visible at higher ray parameters due to spatial gaps in the data. The linear trend from $(12 \mathrm{sec} / \mathrm{deg},-15 \mathrm{sec})$ to $(14 \mathrm{sec} / \mathrm{deg},+8 \mathrm{sec})$ arises from spotty data near $13^{\circ}$. These artifacts make identification of the real tau image difficult. (b) Single-source synthetic stack for model CJF. Compare to Figure 8a. (c) The Mexico data set compared to the northern synthetic stack of (b). Note the poorer agreement, as contrasted to Figure $8 \mathrm{c}$, for $p$ values near 9.5 and $12.8 \mathrm{sec} / \mathrm{deg}$.

beneath the Gulf of California matches the $660 \mathrm{~km}$ depth of GCA'-GCA and not the $650 \mathrm{~km}$ placement of CJF. For $p$ values of 12.7 to $13.0 \mathrm{sec} / \mathrm{deg}$, again GCA'GCA provides a better fit to the $\tau-p$ peaks. GCA'-GCA's lower velocities at 250 $\mathrm{km}$ depth are therefore more compatible with the spreading center data. Thus, even 
fairly subtle structural differences are resolvable using wave field continuation, given high-quality data.

\section{CONCLUSIONS}

A straightforward adaptation of wave field continuation for application to teleseismic body wave data yields useful insights into upper mantle structure. When high-quality, densely sampled digital data are available, this technique provides an inversion which contains all the data in a global format, is relatively easy to implement, and produces an objective estimate of depth resolution as a function of ray parameter. In implementing this method we assume a spatially dense data profile, lateral homogeneity, and coherent source signatures. Since in practice, none of these assumptions may be totally satisfied, some data preparation such as removal of receiver statics and a simple source deconvolution scheme improves the inversion results. Digital array data are essential for obtaining proper spatial sampling. McMechan et al. (1982) demonstrate with data examples that this method works well even when lateral heterogeneity certainly exists.

The limited results presented here by no means exhaust the possible applications of wave field continuation. We have used it as a basis for verifying existing velocity models and for model comparisons, but have not attempted an inversion using this technique alone. Such inversions have been accomplished with refraction data (e.g., Clayton and McMechan, 1981; McMechan et al., 1982). Where the proper data exist, wave field analysis could also provide excellent models, with known error bars, for the lower mantle and the core as well as for other regions of the upper mantle.

\section{ACKNOWLEDGMENTS}

The authors thank Don Anderson, Hiroo Kanamori, and Steve Grand for reviewing the manuscript. This work was supported by Sun Oil Co., National Science Foundation Grant EAR811-5236, and NASA Contract NSG-7610.

\section{REFERENCES}

Backus, G. E. and J. F. Gilbert (1967). Numerical application of a formalism for geophysical inverse problems, Geophys. J. R. Astr. Soc. 13, 247-276.

Backus, G. E. and J. F. Gilbert (1968). The resolving power of gross earth data, Geophys. J. R. Astr. Soc. 16, 169-205.

Backus, G. E. and J. F. Gilbert (1970). Uniqueness in the inversion of inaccurate gross earth data, Phil. Trans. R. Soc. Lond., Ser. A 216, 123-192.

Bessonova, E. N., V. M. Fishman, V. Z. Ryaboyi, and G. A. Sitnikova (1974). The tau method for inversion of travel times. I. Deep seismic sounding data, Geophys. J. R. Astr. Soc. 36, 377-398.

Bessonova, E. N., V. M. Fishman, M. G. Shnirman, G. A. Sitnikova, and L. R. Johnson (1976). The tau method for the inversion of travel times. II. Earthquake data, Geophys. J. R. Astr. Soc. 46, 87-108.

Clayton, R. W. and G. A. McMechan (1981). Inversion of refraction data by wave field continuation, Geophysics 46, 860-868.

England, P. C., B. L. N. Kennett, and M. H. Worthington (1978). A comparison of upper-mantle structure beneath Eurasia and the north Atlantic and Arctic Oceans, Geophys. J. R. Astr. Soc. 54, $575-585$.

Given, J. W. (1984). Inversion of body-wave seismograms for upper mantle structure, Ph.D. Thesis, California Institute of Technology, Pasadena, California, $154 \mathrm{pp}$.

Herrin, E. (1968). 1968 seismological tables for $P$ phases, Bull. Seism. Soc. Am. 58, 1193-1241.

McMechan, G. A. and R. Ottolini (1980). Direct observation of a $p-\tau$ curve in a slant stacked wave field, Bull. Seism. Soc. Am. 70, 775-789.

McMechan, G. A., R. W. Clayton, and W. D. Mooney (1982). Application of wave field continuation to the inversion of refraction data, $J$. Geophys. Res. 87, 927-935. 
Müller, G. (1971). Approximate treatment of elastic body waves in media with spherical symmetry, Geophys. J. R. Astr. Soc. 47, 197-210.

Schultz, P. S. and J. F. Claerbout (1978). Velocity estimation and downward continuation by wavefront synthesis, Geophysics 43, 691-714.

Shaw, P. R. (1983). Waveform inversion of explosion seismology data, Ph.D. Thesis, University of California, San Diego, California, $189 \mathrm{pp}$.

Walck, M. C. (1984a). The $P$-wave upper mantle structure beneath an active spreading center: the Gulf of California, Geophys. J. R. Astr. Soc. 76, 697-723.

Walck, M. C. (1984b). Teleseismic array analysis of upper mantle compressional velocity structure, Ph.D. Thesis, California Institute of Technology, Pasadena, California, $233 \mathrm{pp}$.

Wiggins, R. A. (1969). Monte Carlo inversion of body wave observations, J. Geophys. Res. 74, 31713181.

Wiggins, R. A., G. A. McMechan, and M. N. Toksöz (1973). Range of earth structure nonuniqueness implied by body wave observations, Rev. Geophys. Space Phys. 11, 87-113.

SEISMOLOGICAL LaBORATORY

CALIFORNIA INSTITUTE OF TECHNOLOGY

PASADENA, CALIFORNIA 91125

CONTRIBUTION No. 4063

Manuscript received 15 March 1984 